\title{
Radial Velocity variations of the roAp-star HD122970: new results
}

\author{
A. Gamarova ${ }^{1,3}$, A. Hatzes ${ }^{1}$ and D. Mkrtichian ${ }^{2,3}$ \\ 1 Thüringer Landessternwarte Tautenburg, Sternwarte 5, 07778 Tautenburg, Germany \\ 2 Astrophysical Research Center of the Structure and Evolution of the Cosmos, Sejong University, Seoul \\ 143-747, Korea \\ ${ }^{3}$ Astronomical Observatory, Odessa National University, Shevchenko Park, Odessa 65014, Ukraine
}

\begin{abstract}
In this paper we present new results on the precise relative radial velocity (RV) measurements for the roAp star HD122970. The RV line-by-line frequency analysis revealed the dependence of the pulsational amplitudes on the line strength and a bi-modal phase distribution. These new results can be considered as a significant evidence of the presence of a radial node in the atmosphere of HD122970.
\end{abstract}

The roAp nature of the cool Ap star HD122970 was established by Handler \& Paunzen (1999), who found a main pulsational period of $11.09 \mathrm{~min}(f=129.81427 \mathrm{c} / \mathrm{d})$. Pulsational RV variations were detected by Hatzes et al. (2000). For the present investigation we used 150 high resolution $(R=60,000)$ spectra of HD122970 taken with an iodine cell as a wavelength reference (Hatzes et al., 2000). The RVs were determined over the spectral region between $5000-6300 \AA$. The analysis of the "broad-band" $(\Delta \lambda=10 \AA)$ RV series showed that the signal, corresponding to the main photometric frequency, was present only in several spectral ranges and with different RV amplitudes. Spectral regions dominated by strong lines had amplitudes of around $100 \mathrm{~m} \mathrm{~s}^{-1}$, while regions containing mostly weak lines had amplitudes up to $400 \mathrm{~m} \mathrm{~s}^{-1}$. The RV analysis of individual lines revealed the presence of the pulsational signal only in 21 spectral lines dominated by rare-earth elements. The weaker spectral lines tend to show higher RV amplitudes with the largest amplitude being $1.5 \mathrm{~km} \mathrm{~s}^{-1}$. The lowest pulsational amplitudes tend to occur among relatively strong lines and these are consistent to within the error of the RV amplitude derived using the "broad-band" measurements. Such an amplitude behaviour has been explained as an atmospheric height effect (Kanaan \& Hatzes 1998). The pulsational phase distribution appears to be bi-modal with 6 lines having a phase shifted by about $180^{\circ}$ relative to the mean phase of other lines. This is consistent with the presence of a radial node in the stellar atmosphere of HD 122970, similar to what we found in our study of the roAp star 33 Lib (Mkrtichian et al. 2003) and in $\alpha$ Cir by Baldry et al. (1998). Our results on HD 122970 show the similarities in acoustic structures of roAp stars' atmospheres, giving us the great possibility to investigate the vertical structure of the stellar atmosphere by the acoustic cross-section method (Mkrtichian et al., 2003).

\section{References}

Baldry I. K., Bedding T.R., Viskum M. et al. 1998, MNRAS, 295,33

Handler G., Paunzen E. 1999, A\&AS 135, 57

Hatzes A., Mkrtichian D. \& Kanaan A. 2000, NATO Science Series C. 544, 405

Kanaan A. \& Hatzes A. 1998, ApJ 503, 848

Mkrtichian, D., Hatzes A. \& Kanaan A., MNRAS 2003, in press. 